\section{Hirnmetastasen: stärkere Bestrahlung lohnt sich}

\section{Das am häufigsten angewendete Schema für die Ganzhirnbestrahlung sind 30 Gy in zehn Fraktionen. Kann eine höhere Strahlendosis die lokale Kontrolle verbessern und das Leben der Patienten verlängern?}

$\mathrm{D}$ ie meisten Patienten mit Hirnmetastasen haben nur noch wenige Monate zu leben. Etwas besser ist die Prognose bei höchstens drei Metastasen. Diese Patientengruppe könnte von einer intensiven Behandlung mit Neuro- und Radiochirurgie profitieren, doch für viele kommt wegen der Lage und Größe der Metastasen sowie der Komorbiditäten lediglich eine Ganzhirnbestrahlung infrage. In eine retrospektive Studie wurden nur Patienten mit Hirnmetastasen und günstiger Überlebensprognose (voraussichtlich $78 \%$ nach sechs Monaten) aufgenommen. Verglichen wurden 109 Patienten, die mit der Standarddosis von 30 Gy in zehn Fraktionen bestrahlt wurden, mit 75 Patienten, deren Bestrahlung $40 \mathrm{~Gy}$ in 20 Fraktionen umfasste.
Bei $68 \%$ der Patienten traten innerhalb von neun Monaten Rezidive auf. $28 \%$ der Patienten mit 30-Gy-Bestrahlung und $44 \%$ derer mit 40 -Gy-Bestrahlung waren nach zwölf Monaten rezidivund progressionsfrei (lokale Kontrolle). In der multivariaten Analyse war die Bestrahlung mit 40 Gy signifikant mit besserer lokaler Kontrolle assoziiert ( $\mathrm{p}=$ $0,047)$.

Das 1-Jahres-Überleben betrug $50 \%$ in der 30-Gy- und $61 \%$ in der 40-GyGruppe $(p=0,007)$, das mediane Gesamtüberleben 13 bzw. 17 Monate. Der Unterschied zwischen den beiden Gruppen hinsichtlich des Gesamtüberlebens war auch in der multivariaten Analyse signifikant $(p=0,008)$. In einer Subgruppenanalyse war auch bei Patienten mit weniger radiosensitiven Tumoren die stärkere Bestrahlung der Standarddosis signifikant überlegen: $7 \%$ aus der 30-Gy-Gruppe und $38 \%$ aus der 40-GyGruppe zeigten nach einem Jahr eine lokale Kontrolle $(p=0,031)$. Das 1-JahresGesamtüberleben betrug $40 \%$ bzw. $73 \%$ $(p=0,008)$. Von den Patienten mit anderen Tumortypen erreichten nach einem Jahr $31 \%$ bzw. $45 \%$ eine lokale Kontrolle $(\mathrm{p}=0,26)$, das 1-Jahres-Gesamtüberleben betrug hier $52 \%$ bzw. $59 \%(\mathrm{p}=0,08)$.

Die Häufigkeit von akuten und späten Toxizitäten war unter beiden Therapieregimen vergleichbar.

Fazit: Die Steigerung der Strahlendosis über 30 Gy hinaus erhöht die Überlebenschancen von Patienten mit Hirnmetastasen und relativ günstiger Prognose, am stärksten bei weniger radiosensitiven Tumoren.

Judith Neumaier

Rades D et al. Dose-escalation of whole-brain radiotherapy for brain metastasis in patients with a favorable survival prognosis. Cancer. 2012;118(15):3852-9.

\title{
Chemotherapie als Alternative zur Bestrahlung bei niedriggradigem Gliom
}

\section{Nicht alle Gliome bei Kindern sind resezierbar, eine Bestrahlung birgt in diesem Alter allerdings ein beson- ders hohes Risiko. Was kann eine Chemotherapie leisten?}

$\mathrm{D}$ as ereignisfreie 10-Jahres-Überleben von Kindern mit nicht vollständig reseziertem niedriggradigem Gliom, die bestrahlt wurden, ist mit $74 \%$ relativ hoch. Eine Bestrahlung mit ihren Nebenwirkungen ist aber bei Kindern nur bedingt geeignet - wegen des erhöhten $\mathrm{Ri}$ sikos für Spätfolgen wie z. B. kognitive und endokrine Defizite, Sekundärtumoren u.a. Daher kann man eine Chemotherapie als Alternative in Betracht ziehen. In der vorliegenden Studie wurden zwei Regimes miteinander verglichen.

274 Kinder unter zehn Jahren, bei denen eine Bestrahlung als zu riskant für neurologische Entwicklungsstörungen galt, wurden aufgenommen. Alle litten an einem progredienten oder residualen Gliom WHO-Grad-1 oder -2. Außer der Operation war zuvor keine Behandlung erfolgt. Im Rahmen der Studie erhielten die Kinder randomisiert Carboplatin/ Vincristin (CV) oder Thioguanin/Procarbazin/Lomustin/Vincristin (TPCV).

Bei 151 Kindern kam es zum Therapieversagen, 38 starben. Zwei Patienten in der TPCV-Gruppe entwickelten sekundäre Malignome. Im CV-Arm lag das ereignisfreie 5-Jahres-Überleben bei 39\%, im TPCV-Arm bei $52 \%$. Der Unterschied war im stratifizierten Log-rank-Test nicht signifikant $(\mathrm{p}=0,10)$, aber in einer $\mathrm{zu}$ Studienbeginn nicht geplanten Analyse nach dem Cure-Model ( $\mathrm{p}=0,007)$. Das 5-Jahres-Gesamtüberleben betrug im CV-Arm 86\%, im TPCV-Arm 87\%.

In der multivariaten Analyse erwiesen sich zwei Faktoren als unabhängige Prädiktoren für ereignisfreies und Gesamtüberleben: Alter und Tumorgröße. Kinder unter einem Jahr hatten ein 3,4-mal so hohes relatives Risiko für Progression bzw. Rezidiv wie Kinder über fünf Jahren. Bei Kindern mit Resttumoren unter $3 \mathrm{~cm}^{2}$ betrug das relative Risiko für eine Progression bzw. ein Rezidiv nur etwa zwei Drittel $(0,65)$ des Risikos bei Kindern mit größeren Resttumoren. Für das Gesamtüberleben war neben diesen beiden Faktoren auch die Tumorlokalisation von Bedeutung: Ein Tumor im Thalamus war mit einem schlechteren Gesamtüberleben assoziiert.

Abgesehen von den allergischen Reaktionen gegenüber Carboplatin verursachte das TPCV-Regime etwas mehr Toxizitäten.

Fazit: Bei Kindern mit nicht vollständig reseziertem niedriggradigen Gliom ist TPCV vermutlich etwas wirksamer als CV. Unterschiede bei den Toxizitäten könnten die Therapieentscheidung beeinflussen.

Judith Neumaier

Ater JL et al. Randomized study of two chemotherapy regimens for treatment of low-grade glioma in young children: A report from the Children's Oncology Group. J Clin Oncol. 2012;30(21):2641-7. 